\title{
Modelling and assessing the impact of the DSO remuneration strategy on its interaction with electricity users
}

\author{
Miguel Manuel de Villena ${ }^{\mathrm{a}, *}$, Axel Gautier ${ }^{\mathrm{b}}$, Damien Ernst ${ }^{\mathrm{a}}$, Mevludin Glavic ${ }^{\mathrm{a}}$, \\ Raphael Fonteneau ${ }^{a}$ \\ ${ }^{a}$ Department of Electrical Engineering and Computer Science, University of Liège, Belgium \\ ${ }^{\mathrm{b}}$ Department of Economics, University of Liège, Belgium
}

\section{A R T I C L E I N F O}

\section{Keywords:}

Distribution system operator

Distributed electricity generation resources

Distribution tariffs

Regulation

Remuneration strategy

\begin{abstract}
A B S T R A C T
This paper presents a simulation-based methodology for assessing the impact of employing different distribution system operator's remuneration strategies on the economic sustainability of electrical distribution systems. The proposed methodology accounts for the uncertainties posed by the integration of distributed electricity generation resources, and the roll out of smart meters. The different remuneration strategies analysed in this paper include notably new distribution tariffs based on individual peak power consumption and time-dependent rates that are contingent on the time of energy consumption, both requiring smart meters to work. The distributed electricity generation resources are modelled through an optimisation framework and an investment decision process that gradually deploys household photovoltaic installations depending on their profitability and the electricity charges, including the distribution rates. The impact of the distribution system operator's remuneration strategy is measured by an accurate modelling of the remuneration mechanism of this entity, which can adapt to various distribution tariff designs. We analyse this impact over a discrete time horizon. Our methodology is illustrated with several examples of distribution tariffs including old -based on energy consumption or on per-connection fees- as well as new -based on power consumption or time-of use fees- designs. Finally, we provide a comprehensive sensitivity analysis of the proposed simulation environment to the main parameters of the methodology.
\end{abstract}

\section{Introduction}

One of the central objectives of the energy transition process is to progressively shift from fossil fuel-based power generation to lowcarbon, renewable alternatives [1]. The integration of distributed electricity generation resources (DERs) has been deemed a key enabler of a successful energy transition and thereby, DERs are typically promoted by means of various incentive mechanisms, which vary from region to region [2]. These incentive mechanisms, nonetheless, may sometimes have unforeseen and harmful effects on the electricity distribution sector, which are difficult to identify a priori. Indeed, since the distribution networks are not technically and administratively designed to absorb large amounts of distributed generation [3], the incorporation of DER may cause both severe technical disruption [4] and regulatory challenges [5]. This paper proposes a methodology to test novel regulatory frameworks promoting the integration of residential DER, usually composed of solar photovoltaic (PV) panels and/or batteries, evaluate their effectiveness, and identify their shortcomings. More precisely, assuming that a constant part of the distribution system operator (DSO) costs must be recovered through the distribution charges to distribution network users, we investigate how business models exploiting behindthe-meter devices to reduce electricity bills may impact on the remuneration mechanisms of DSOs.

\footnotetext{
* Corresponding author.

E-mail addresses: mvillena@uliege.be (M. Manuel de Villena), agautier@uliege.be (A. Gautier), dernst@uliege.be (D. Ernst), mevludin.glavic@uliege.be (M. Glavic), raphael.fonteneau@uliege.be (R. Fonteneau).
} 


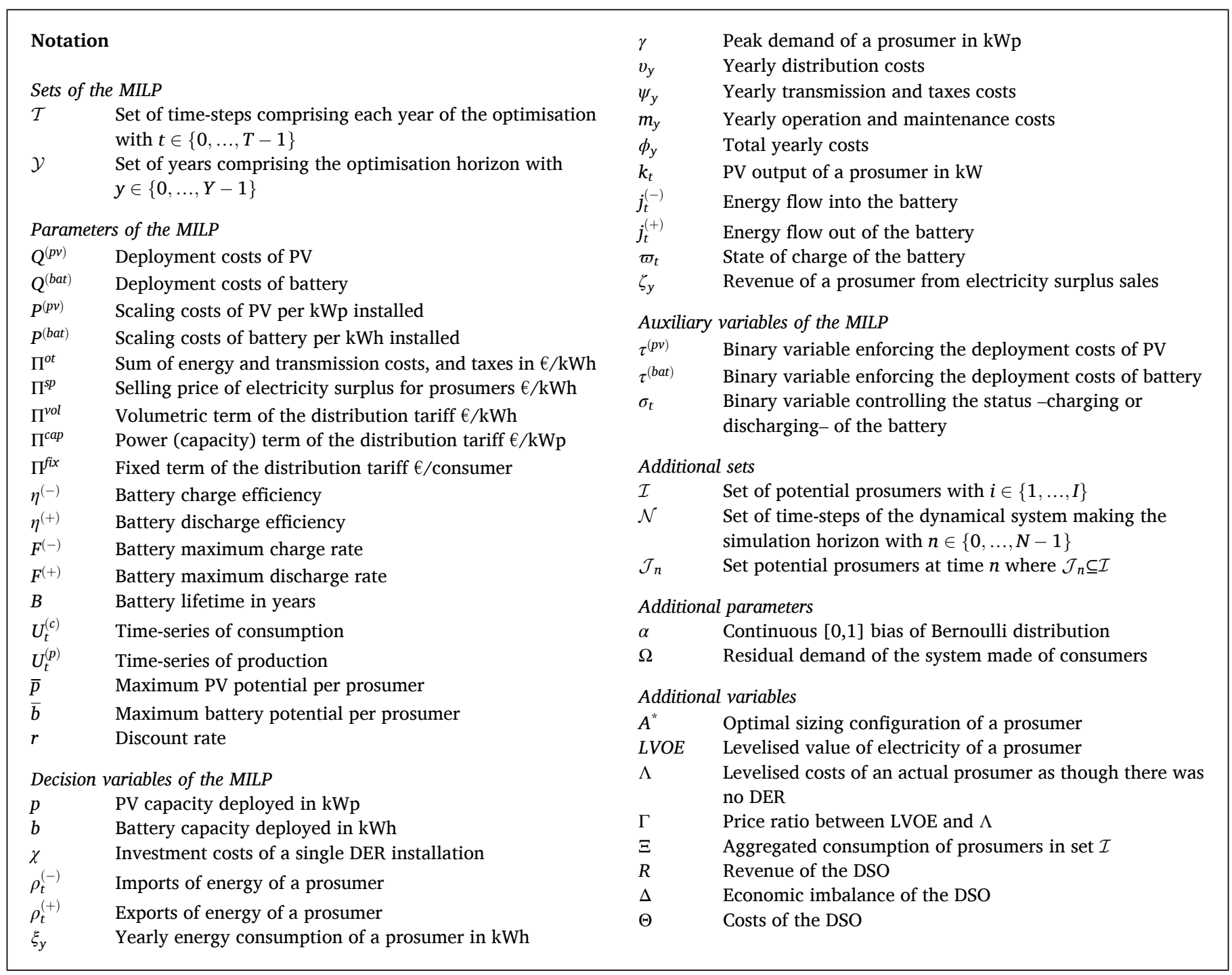

Previous studies on the topic suggest that the integration of DERs into the distribution networks induce changes in the way in which the distribution network is used, challenging its normal operation. Such changes, according to [6], are region independent, therefore representing a worldwide dilemma, and raise the question of how to distribute the costs in these new distribution systems. The authors of this report review the distribution tariff structures of several countries/regions, ${ }^{1}$ and simulate their effects through notional households. The authors introduce several notions of fairness, highlighting the importance of finding the right scheme to deter an unfair allocation of distribution costs among final customers, and stressing that the fairness of the scheme depends on the interpretation of this concept. Another report, this time centred in Australia, discusses distribution tariff reforms in Victoria's distribution network [7]. The authors outline

\footnotetext{
1 This report ([6]) analyses four European Union Member States: Italy, Portugal, Romania, and The Netherlands; one European Economic Area State: Norway, and one state outside European jurisdiction: the State of California in the US. The distribution tariff schemes in each of these examples is different: Italy - energy, power, and fixed components, with an increasing block tariff; Portugal - energy and power components, with a time-of-use basis; Romania energy component; The Netherlands - power and fixed components; Norway energy and fixed components; California - energy and fixed components, with an increasing block tariff and a time-of-use basis.
}

different tariff options toward distinct objectives, making use of the principles of simplicity, efficiency, adaptability, affordability, and equity. Similar principles are suggested in other research articles such as $[8,9]$. The works presented in $[10,11]$ indicate that, under certain regulatory frameworks designing the DSO remuneration strategies, the deployment of DER, such as household PV units, may be responsible for a non-negligible increase in the distribution component of the overall retail price of electricity (the latter typically including energy generation costs, transmission costs, distribution costs, and taxes). In particular, [10] suggests reviewing tariff designs based on volumetric charges with single metering, arguing that these designs are not cost reflective and potentially lead to cross-subsidies, proposing bi-directional metering as an alternative. To add to the previous, the authors in [11] make the comparison of a single metering mechanism (net-metering) with a dual one (net-purchasing), advocating the use of the latter in order to create more accurate price signals to synchronise consumption and production and to avoid cross-subsidisation from consumers to prosumers. Furthermore, the authors in [12] show, with empirical data, that in a setting where the distribution charges to the consumers are predominantly volumetric (i.e. in $€ / \mathrm{kWh}$ ), an increase in the distribution tariff leads to a corresponding increase in household PV deployment. The combination of these effects can result in a potentially disrupting phenomenon known as the death spiral of the utility.

This concept is introduced in [13], where it is analysed in depth and 
tentative solutions from the DSO stand point are proposed to mitigate its potentially harming effects (e.g., approval of new rate-making practices or support for new business models). In another work, [14], the author states that an inadequate flat-rate tariff design in Queensland, Australia has led to network price increases of $112 \%$ owing to a death-spiralrelated problem. The death spiral takes place in two stages: (1) distribution tariffs increase due to the deployment of DER (DSOs struggle to recover their costs and must increase the distribution tariffs), and (2) higher distribution tariffs induce the proliferation of DER installations (or other types of response from final customers to mitigate on their end the tariff increase). Should this phenomenon proceed unchecked for some time, an uncontrolled increase in distribution tariffs may occur, in which the extra financial burden resulting from higher tariffs is mostly met by the users who have not deployed DER, and who are thus more exposed to price fluctuations, as shown in $[11,15]$. The latter work proposes a stylised framework assessing the costs for consumers and prosumers after the deployment of DER installations, in a setting where net-metering is employed, quantifying the difference in costs. This difference in costs may result in cross-subsidies from traditional consumers to DER owners, as shown in $[10,16]$. In [16], the authors suggest a connection between the self-consumption rate (i.e., the proportion of a prosumer's consumption covered by their own DER installation) and the level of cross-subsidies from consumers to prosumers, in a study focused on France. A similar observation is made across the Atlantic in [17], where different distribution tariff designs in Texas, US, are assessed, reporting on their impact on the distribution network as a function of the level of cross-subsidisation -proxy for unfairness according to the authors- they induce.

To cope with these problems, several DSO remuneration strategies have been proposed and analysed - strategies that better reflect the costs of the electricity distribution, and induce electricity rates that serve as efficient signals for the users of the distribution network, as explained and recommended in [18]. The challenges created by the integration of DER are qualitatively analysed in [19], where the authors recommend regulatory improvements on the remuneration mechanism of DSOs, taking into account the cost-reflectivity principle. In particular, they recommend the use of incentive regulation based on price or revenue caps rather than rate of return regulation, where DSOs are allowed to keep any efficiency gains from efficient DER integration. Other costreflective strategies are analysed in [20], where the transition from a distribution tariff based on average costs to a cost-causation tariff that looks into time and location to determine the costs (via e.g., coincident peak) is analysed. The authors ultimately show the importance of breaking down the different effects a change in distribution tariff may induce, to quantitatively understand their foreseeable impacts. Hence, quantitatively assessing the effectiveness and potential pitfalls of novel DSO remuneration strategies is essential, and simulation-based techniques can be invoked to test them in various technological and regulatory settings.

We can find several examples in the literature where the authors have made use of different simulation-based techniques to attain similar goals. The authors in [21] develop an framework to establish the remuneration mechanisms of DSOs. Such a framework lays out a global remuneration scheme to compute the distribution tariff, which is based on a revenue-limitation scheme that considers initial distribution costs, annual market increases, and efficiency gains. Several works have made use of agent-based modelling to analyse this type of problem. For instance, in [22], a simulation approach based on multi-agent modelling is developed to analyse the impact of the integration of renewable resources (wind in this case) on the efficient use of the transmission system in Québec, Canada. Similarly, in [23], a multi-agent-based model is developed and applied to study the integration of distributed generation units where the agents are the DER installations. This tool is employed to help ensure the power system balance control in Hungary. The previous two works focus on control problems but show the suitability of these frameworks to model renewable resources and, in particular, DER integration. In [24], a quantitative approach is presented to assess distribution network performances when presented with incentive-based regulation. These performances are measured with and without DERs, and serve to guide DSO investments as well as to quantify the impact of incentive regulation on these investments. This topic is also dealt with in [25], where a method for regulators to find the right incentive scheme for distributed generation is exposed. The proposed method is based on a multi-objective optimisation problem that provides pareto-optimal solutions to the decision to invest in DERs from the investor (maximisation of the net present value) and the DSO (maximisation of the net present value derived from the provided incentives) perspectives. In [26], an active distribution network is simulated by means of multi-agent systembased modelling, using cooperative agents representing different loading scenarios. A non-cooperative game is proposed in [27], where different tariff structures are evaluated, and their impacts on the electricity users are studied. This work is further developed in [28], where the authors introduce three types of fee to design the distribution tariff: energy, power and fixed; considering prospective, in additional to sunk costs, to set the tariff level. In [9], the design of cost-reflective distribution tariffs is addressed, introducing a model in which users can react to high distribution charges by deploying fix-sized DER installations in order to reduce their electricity bills. The impact of regulation on the willingness of DSOs to integrate distributed generation is addressed in [29], where a method is proposed and applied to different case studies. Finally, [30] introduces a stylised set-up where two different metering systems (net-metering and net-billing) are analysed in their ability to promote the deployment of DER. In the latter work, the impact of such metering systems on the consumers in the distribution network and on the electricity prices is studied, concluding that the death spiral of the utility might be a potential issue, in particular in the net-metering case which can be considered as an incentive mechanism on its own. All these works deal with simulation-based analysis of the relation between DSO remuneration strategies, DER integration, and impact on distribution networks.

Building upon the existing literature, this paper introduces a simulation-based computational tool that enables the modelling and study of the multi-agent system dynamics resulting from interactions between the agents of a distribution network, namely the distribution network users and the DSO. At every time-step, agents may either stay idle or perform a pre-defined action: the distribution network users can deploy optimally sized PV installations with or without batteries aiming at minimising their electricity bills, whereas the DSO can adjust the distribution tariff in order to collect sufficient revenue so as to break even. Hence, the present paper adds to the literature by explicitly modelling the action-reaction dynamics of agents under various tariff structures, thereby allowing to represent the system evolution over time and estimate the short-to-middle-run effects of specific pieces of regulation on aforementioned distribution network attributes.

In the remainder of this paper, Section 2 establishes the concrete contributions of our work. Section 3 provides an introductory overview of the simulation-based approach. Section 4 details the underpinning mathematical models. Section 5 illustrates the methodology considering various regulatory frameworks and DSO remuneration strategies, and tests the limits of the simulation-based computational tool by introducing an extensive sensitivity analysis of the main parameters of the model. Finally, Section 6 concludes the paper.

\section{Contributions}

Our approach adds to the previous works, notably including [30] by:

- Mathematically formalising a sizing tool which is used to optimally size DER installations.

- Mathematically formalising an investment decision process for modelling the adoption and deployment of DER installations based on the cost-efficiency and profitability of the installation. 
- Modelling, in a realistic fashion, the non-linear investment costs of deploying DER installations by making use of a continuous piecewise approximation which is more accurate than the traditional approach whilst being computationally efficient.

- Mathematically formalising the remuneration mechanism of DSOs that determines the economic balance (or imbalance) of the DSO, which depends on the distribution tariff and the DSO costs - this mechanism must take into account all possible distribution tariff structures, i.e. based on units of energy consumed, units of power consumed, or type of access point to the distribution network.

- Introducing the concept of levelised value of electricity (LVOE) as an extension of the traditional levelised cost of electricity (LCOE) to take into account not only the costs of DER installations, but also potential revenue via electricity sales - the LVOE is then used both as the objective function of a minimisation problem and as a metric on which to report.

The simulation environment presented in our work requires a tariff design as input, which is typically set by the regulator. In previous works (such as [30]), these designs were limited to mechanical meters, therefore only volumetric and fixed fees were possible. In this paper we assume full roll out of smart meters, opening the door to new tariff designs. Thus, in addition to the previous, we expand the current literature by introducing:

- Capacity fees by which the DSO charges the users depending on the power they draw from the distribution network.

- Time-of-use (ToU) fees that are time varying, i.e. the costs for the users depend on the time of the day.

We thus provide one single simulation environment which can assess, in a realistic fashion, the impact of all the different tariff designs (volume, fixed, capacity, ToU) on a detailed investment decision process where prosumers are accurately modelled through an optimisation framework, taking into account a coherent representation of the DSO remuneration mechanism.

\section{Simulation configuration}

The proposed methodology relies on a multi-agent system formalisation in which the agents (i.e. consumers, potential prosumers, actual prosumers, and the DSO) interact with each other within a given set of rules characterising a technical and a regulatory framework. Through the agents' interactions over time, the use of the distribution network changes, and so does the distribution tariff. Then, by tracking the actions of agents across a provided simulation horizon, we can determine trajectories of prices over such a horizon. By using this principle utilising various starting conditions, we may estimate the different use changes in the distribution network those starting conditions induce.

Each type of agent interacts in a different way:

- Consumers are passive agents who simply consume electricity from the distribution network according to their demand profiles. They cannot become prosumers owing to technical or economic constraints and are modelled through their electricity demand.

- Potential prosumers are agents who may deploy an optimally sized DER installation, turning into actual prosumers; the decision to deploy such an installation depends on its cost-efficiency when compared to the retail price of electricity. After the comparison is computed, a probabilistic investment decision process is laid out to determine whether a given potential prosumer becomes an actual prosumer.

- Actual prosumers are passive agents who consume and produce electricity from the distribution network according to their demand and production profiles. Such profiles are established only when potential prosumers become actual prosumers, therefore reflecting the after-the-meter consumption or production accounting for the deployed DER installations.

- The DSO manages the distribution network, incurring certain costs in this role. Through its remuneration mechanism, the DSO collects charges for the use of the distribution network by the three types of user (consumers, potential prosumers, and actual prosumers), and is entitled to adjust the distribution tariff so that it recovers the totality of its costs, breaking-even.

Through the agents' interactions over time, it is possible to determine the evolution of the proportions of consumers, potential prosumers, and actual prosumers, as well as the evolution of distribution tariff and electricity exchanges over a provided simulation horizon. The simulation starts with a pool of potential prosumers who may become actual prosumers during the simulation, relying less on the distribution network. The DSO, expecting to collect a certain level of charges from these potential prosumers, in fact collects a different level since the consumption behaviour of actual prosumers is different to that of potential prosumers. As a result, the DSO may adjust the distribution tariff to adapt to the new situation. The full modelling of these agents as well as the simulation procedure is detailed in the following section.

\section{Modelling and problem formalisation}

In this section, we present the models used in our simulation-based computational tool. We start by describing the set of rules defining the technical and regulatory frameworks and then, we formalise the different agents and their interaction mechanisms.

\subsection{Rules defining the technical and regulatory frameworks}

These rules define the playing field for agents to interact. A real-life playing field includes many rules, which may not all be relevant to our modelling. Against this backdrop, we identified and selected a sub-set of rules capturing key drivers for DER deployment: tariff design and technology costs.

\subsubsection{Tariff design}

This sub-set of rules defines the structure of the distribution costs charged to the users of the distribution network. In our work we consider that the distribution tariff might be based on volume of energy drawn from the grid charged in $€ / \mathrm{kWh}$, power drawn charged in $€ / \mathrm{kWp}$, or connection point charged in $€ /$ user. The amount of money charged by the DSO for its services over a given billing period is obtained as a weighted sum of those fees, whose respective proportions are regulated. To design a tariff, it is possible to use any combination of these fees.

In addition, in our simulation-based approach we introduce ToU tariffs by setting different time-dependent price levels. Those levels are applied to volume fees. Accordingly, under a ToU tariff, the volume fee of the distribution tariff will comprise several sub-fees, depending on the time of consumption.

\subsubsection{Technology costs}

This sub-set of rules has an impact on the investment costs of prosumers. In our work, we divide these costs in two.

- Deployment costs are charges that depend on whether the DER installation is deployed or not. They represent the costs of installation, including the PV, inverter, and (if any) batteries.

- Scaling costs are the charges depending on the scale of the installation. We assume these costs to be linearly dependent on the size of the installation, therefore on the total deployed capacity of PV and battery.

These are therefore non-linear costs that we model using a piecewise linear approximation where the two terms are introduced (see 4.3 for 
more details). Furthermore, we assume these two components will linearly decrease over time. This means that the technology will be more expensive at the beginning of the simulated period than at the end. In this sense, prosumers who deploy DER later in time will pay less for their installations.

\subsection{Users}

Users are divided into three groups: (i) consumers, (ii) potential prosumers, and (iii) actual prosumers. The consumers group comprises users who will not deploy a DER installation due to economic or technical constraints. Their aggregated demand (also known as the residual demand of the distribution network) is used in the simulation. We define potential prosumers as all the users who may deploy a DER installation, provided that the conditions are favourable. Potential prosumers are, initially, consumers importing electricity from the grid to cover their demand. Then, as the simulation proceeds over time, the number of potential prosumers may decrease as they elect to invest in and progressively deploy optimally sized DER installations, effectively turning into actual prosumers. Finally, actual prosumers are able to import and export electricity from and into the distribution network.

To model the interactions of potential and actual prosumers, we make use of an optimisation framework. We formulate this optimisation as a mixed integer linear problem (MILP) aimed at minimising the levelised value of electricity (LVOE) of a DER installation. We introduce the concept of LVOE -whose formulation can be found in Section 4.3- as an extension of the traditional levelised cost of electricity (LCOE). The difference between these two concepts is that whilst the LCOE can only account for the costs incurred by the DER installation, the LVOE can take into consideration both costs and revenue (for instance revenue obtained from electricity sold). Adding the dimension of revenue was not needed in the past, where net-metering was predominant, since, with this system, the revenue are implicitly taken into account. However, with the introduction of other mechanisms such as net-billing, where imports and exports are measured separately, the concept of LCOE falls short in accurately describing the dynamics of prosumers, being necessary to introduce the LVOE to explicitly integrate the revenue. The LCOE is therefore computed as costs divided by demand, whereas the LVOE is expressed as costs minus revenue divided by demand (in all cases an annual discount rate is applied to costs, revenue, and demand). Hence, the LVOE provides an indication of the net economic gain of potential prosumers, should they become actual prosumers. Moreover, by comparing the LVOE with the electricity costs without DER we can compute the probabilistic investment decision process of potential prosumers becoming actual prosumers. Both the MILP and the investment decision process are presented in the remainder of this section.

\subsection{Optimisation framework formalisation}

For every individual potential prosumer, this optimisation program is used to compute the electricity trades (imports and exports), the minimised LVOE, and the optimal sizing configuration of the DER installation leading to the minimum LVOE. Hence, both sizing and operation are optimised under a perfect forecast assumption. The optimisation horizon is set to $Y \in \mathbb{N}$ years, where $\mathcal{Y}=\{0, \ldots, Y-1\}$ (not to be confused with the simulation horizon, which will be presented later in Section 4.4). Each year is divided into $T$ time-steps. Let $\mathcal{T}=$ $\{0, \ldots, T-1\}$, where $T=8760$ represents a time discretisation of one year in hours. The MILP requires several parameters as inputs; these parameters are constant over the simulation horizon $Y$ since they do not evolve from year to year of the optimisation (note that some of them will evolve over the simulation horizon, see Section 4.4). Let $G$ denote a 4 tuple gathering these inputs:

$$
\begin{gathered}
G=(P, \Pi, H, \mathbf{U}) \in \mathcal{G}, \text { with } \\
\mathcal{G} \subset\left(\mathbb{R}_{+}^{4}\right) \times\left(\mathbb{R}_{+}^{5}\right) \times\left(\mathbb{R}_{+}^{5}\right) \times\left(\mathbb{R}_{+}^{2}\right)^{T},
\end{gathered}
$$

where:

- $P=\left(Q^{(p v)}, Q^{(b a t)}, P^{(p v)}, P^{(b a t)}\right)$ represent the deployment costs of PV $\left(Q^{(p v)}\right)$ and batteries $\left(Q^{(b a t)}\right)$, as well as the scaling costs of PV per $\operatorname{kWp}\left(P^{(p v)}\right)$ and batteries per kWh $\left(P^{(b a t)}\right)$. See Section 4.1.2 for a reminder on deployment and scaling costs.

- $\Pi=\left(\Pi^{(o t)}, \Pi^{(s p)}, \Pi^{(v o l)}, \Pi^{(c a p)}, \Pi^{(f i x)}\right)$ are price signals. $\Pi^{(o t)}$ is the aggregation of energy costs, transmission costs, and taxes, in $€ / \mathrm{kWh}$. $\Pi^{(s p)}$ corresponds to the price at which prosumers sell the electricity in $€ / \mathrm{kWh} . \Pi^{(v o l)}$ is the volumetric term of the distribution tariff in $€ / \mathrm{kWh} . \Pi^{(c a p)}$ represents the capacity term of the distribution tariff in $€ / \mathrm{kWp} . \Pi^{(f i x)}$ represents a fixed charge to be paid by every user connected to the distribution network, in $€$. In the case of ToU tariffs, $\Pi^{(v o l)}$ and/or $\Pi^{(c a p)}$ will present different levels depending on the time of consumption.

- $H=\left(\eta^{(-)}, \eta^{(+)}, F^{(-)}, F^{(+)}, B\right)$ defines the battery parameters. $\eta^{(-)}$is the charge efficiency. $\eta^{(+)}$is the discharge efficiency. $F^{(-)}$represents the maximum charge rate. $F^{(+)}$stands for the maximum discharge rate. Finally $B$ is the battery lifetime in years $(B>0)$.

- $\mathbf{U}=\left\{\left(U_{t}^{(c)}, U_{t}^{(p)}\right)\right\}_{t=0}^{T-1}$ is a time-series of pairs representing the potential prosumer consumption profile $\left(U_{t}^{(c)}\right)_{t=0 \ldots T-1}$ (in terms of hourly energy consumption), and the solar load factor $\left(U_{t}^{(p)}\right)_{t=0 \ldots T-1}$ (in \%), respectively.

Let $\mathcal{A}=\{(p, b): p \in[0, \bar{p}] ; b \in[0, \bar{b}]\}$ denote the space of sizing variables containing: $\mathrm{PV}$ size $(p)$ in $\mathrm{kWp}$, battery size $(b)$ in $\mathrm{kWh}$; with $\bar{p}$, and $\bar{b}$ being parameters denoting the upper bounds on PV and battery capacities, respectively. Furthermore, let $\tau^{(p v)}$ and $\tau^{(b a t)}$ denote binary variables enforcing the deployment costs when either PV or batteries are installed. Finally, let $\chi$ represent the investment costs of PV and batteries, which are linearised by means of a piecewise affine function, and are dependent on the sizing variables $A \in \mathcal{A}$.

$\chi=p \cdot P^{(p v)}+\frac{Y}{B} \cdot b \cdot P^{(b a t)}+\tau^{(p v)} \cdot Q^{(p v)}+\tau^{(b a t)} \cdot Q^{(b a t)}$,

where the control of the binary variables $\tau^{(p v)}$ and $\tau^{(b a t)}$ is given by:

$p \leqslant \bar{p} \cdot \tau^{(p v)}$

$b \leqslant \bar{b} \cdot \tau^{(b a t)}$.

The yearly costs incurred by a prosumer are represented by $\phi_{y}$, and computed by means of the following equation:

$\phi_{y}=v_{y}+\psi_{y}+m_{y} \quad \forall y \in \mathcal{Y}$,

where $v_{y}$ represents the yearly electricity distribution costs, computed according to Eq. (5). $\psi_{y}$ stands for the yearly costs of electricity not related to distribution costs, i.e. transmission and energy costs, computed using Eq. (6). $m_{y}$ are the costs of operating and maintaining the DER installation; these costs are computed as in [31], following Eq. (7).

$v_{y}=\xi_{y} \cdot \Pi^{(v o l)}+\gamma \cdot \Pi^{(c a p)}+\Pi^{(f i x)} \quad \forall y \in \mathcal{Y}$,

$\psi_{y}=\xi_{y} \cdot \Pi^{(o t)} \quad \forall y \in \mathcal{Y}$,

$m_{y}=\frac{1}{200} \cdot p+\frac{1}{100} \cdot b \quad \forall y \in \mathcal{Y}$,

in these equations, $\xi_{y}$ and $\gamma$ represent the yearly consumption and the peak demand of a prosumer, respectively. They are computed as follows: 
$\xi_{y}=\sum_{t=0}^{T-1} \rho_{t}^{(-)} \quad \forall y \in \mathcal{Y}$,

$\gamma=\max \left\{\rho_{t}^{(-)}: t=0, \ldots, T-1\right\}$,

where $\rho_{t}^{(-)}$are the hourly imports of a prosumer. To define the energy balance we need to define: the exports of electricity $\rho_{t}^{(+)}$, the PV output of each DER $k_{t}$ (Eq. 10), and the energy flows into and out of the battery $j_{t}^{(-)}$and $j_{t}^{(+)}$respectively (Eqs. (11)-(14)).

$k_{t}=p \cdot U_{t}^{(p)} \quad \forall t \in \mathcal{T}$,

$j_{t}^{(-)} \leqslant b \cdot \frac{1}{F^{(-)}} \quad \forall t \in \mathcal{T}$,

$j_{t}^{(+)} \leqslant b \cdot \frac{1}{F^{(+)}} \quad \forall t \in \mathcal{T}$,

$j_{t}^{(-)} \leqslant \bar{b} \cdot \sigma_{t} \quad \forall t \in \mathcal{T}$,

$j_{t}^{(+)} \leqslant \bar{b} \cdot\left(1-\sigma_{t}\right) \quad \forall t \in \mathcal{T}$.

In these equations, $\sigma_{t}$ is a binary variable taking a value of 1 when the battery is charging, and 0 if it is discharging. Then, the energy balance is given by:

$U_{t}^{(c)}+\rho_{t}^{(+)}+j_{t}^{(+)}=k_{t}+\rho_{t}^{(-)}+j_{t}^{(-)} \quad \forall t \in \mathcal{T}$.

The last variable of our model is the state of charge of the battery, $\varpi_{t}$.

$\varpi_{t} \leqslant b \quad \forall t \in \mathcal{T}$,

$\varpi_{t}=\left\{\begin{array}{c}\varpi_{t-1}-\frac{j_{t}^{(+)}}{\eta^{(+)}}+j_{t}^{(-)} \cdot \eta^{(-)}, \forall t \in \mathcal{T} \backslash\{0\} \\ 0 \quad \text { if } \quad t=0\end{array}\right.$

Finally, let LVOE denote the general objective function of the MILP that represents the levelised value of electricity. This function will be minimised when the MILP is instantiated and solved. It is a mapping from $(\mathcal{G} \times \mathcal{A})$ to $\mathbb{R}$. For a given pair $(G, A) \in(\mathcal{G}, \mathcal{A}), \operatorname{LVOE}(G, A)$ is defined as follows:

$\operatorname{LVOE}(G, A)=\frac{\chi+\sum_{y=0}^{Y-1} \frac{\phi_{y}-\zeta_{y}}{(1+r)^{y}}}{\sum_{y=0}^{Y-1} \frac{\sum_{t=0}^{T-1} U_{t}^{(c)}}{(1+r)^{y}}}$,

where $\zeta_{y}$ is the revenue of the prosumer from electricity sales, and $r$ is the discount rate. By subtracting $\zeta_{y}$ from the operational costs $\phi_{y}$, we compute the actual value offered by the DER installation (LVOE), instead of simply its levelised cost. This term depends on the total amount of energy exported to the grid and on the selling price of electricity at which the prosumers can sell the electricity to the grid, as expressed in Eq. (19):

$\zeta_{y}=\sum_{t=0}^{T-1} \rho_{t}^{(+)} \cdot \Pi^{(s p)} \quad \forall y \in \mathcal{Y}$.

From this MILP we extract the values of several variables to be used later on. They are the optimal sizing variables $p$ and $b$; the yearly consumption $\xi_{y}$ the yearly peak demand $\gamma$.

\subsection{Expanding the optimisation framework to multiple time-steps and prosumers}

At the heart of the simulation-based approach lies a discrete-time dynamical process computing the evolution of a set of indicators. Let $n \in$ $\mathcal{N}$ denote the discrete-time variable used to refer to the iterations of this dynamical process, where $\mathcal{N}=\{0, \ldots, N-1\}$, and $N \in \mathbb{N}$ is the time horizon. Furthermore, to represent the diversity of users, we introduce a set of $I \in \mathbb{N}$ potential prosumers, with $\mathcal{I}=\{1, \ldots, I\}$. At every iteration $n$, each potential prosumer $i \in \mathcal{I}$ is characterised by a time series of pairs $\mathbf{U}_{i, n}=\left\{\left(U_{i, n, t}^{(d)}, U_{i, n, t}^{(p)}\right)\right\}_{t=0}^{T-1}$. Therefore, at every iteration $n$, and for every user $i$, we can define:

$G_{i, n}=\left(P_{n}, \Pi_{i, n}, H_{n}, \mathbf{U}_{i, n}\right) \quad \forall(i, n) \in \mathcal{I} \times \mathcal{N}$,

where $P_{n}$ and $H_{n}$ do not depend on $i$ since they refer to technology costs and technical characteristics, assumed identical for all users. Consequently, we define $\widehat{L V O E}_{G_{i, n}}$ as the minimum value of the objective function, subject to the previous constraints:

$\widehat{L V O} E_{G_{i, n}}=\min _{\substack{A \in \mathcal{A} \\ \text { s.t. }(1)-(19)}} \operatorname{LVOE}\left(G_{i, n}, A\right)$

Furthermore, the optimal sizing configuration is written as:

$A_{G_{i, n}}^{*} \in \underset{A \in A}{\arg \min } \operatorname{LVOE}\left(G_{i, n}, A\right)$ $A \in \mathcal{A}$
s.t. $(1)-(19)$

\subsection{Investment decision process}

From one time-step in the simulation horizon to the next, we compute the transition from potential to actual prosumer. For each potential prosumer, the $\widehat{L V O E} G_{i, n}$ is compared to the levelised cost without DER (denoted by $\Lambda_{i, n}$ ). The outcome of this comparison defines whether or not a transition occurs. Let $\mathcal{J}_{n} \subseteq \mathcal{I}$ denote the set of potential prosumers at time $n$. Initially, $\left|\mathcal{J}_{0}\right|=|\mathcal{I}|$. Assuming that prosumers cannot turn back into consumers, one has $\forall n \in\{0, \ldots, N-1\}$, $\left|\mathcal{J}_{n}\right| \leqslant\left|\mathcal{J}_{n-1}\right|$. Then, the costs $\Lambda_{i, n}$ are calculated as follows:

$\Lambda_{i, n}=\Pi_{n}^{(o t)}+\Pi_{n}^{(v o l)}+\frac{\gamma_{i, n}^{(o)} \cdot \Pi_{i, n}^{(c a p)}+\Pi_{n}^{(f i x)}}{\sum_{t=0}^{T-1} U_{i, n, t}^{(c)}} \quad \forall(i, n) \in \mathcal{J}_{n} \times \mathcal{N}$,

where $\gamma_{i, n}^{(o)}$ is the original peak demand of the user. Then, a price ratio $\Gamma_{i, n}$ can be computed as:

$\Gamma_{i, n}=\frac{\widehat{L O O} E_{G_{i, n}}}{\Lambda_{i, n}} \quad \forall(i, n) \in \mathcal{J}_{n} \times \mathcal{N}$.

In this last equation, $\Lambda_{i, n}$ is strictly positive provided that the demand of the user and the electricity prices are strictly positive. $\Gamma_{i, n}$ will therefore adopt a value between 0 and 1 , since $L \widehat{V O} E_{G_{i, n}}$ cannot be greater than $\Lambda_{i, n}$, by design of the optimisation problem. To establish whether a consumer will decide to deploy a DER installation, we make use of a Bernoulli random variable whose parameter $p_{i, n}$ is a function of $\Gamma_{i, n}$.

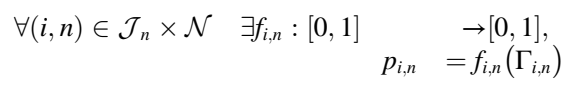

For simplicity, in the following we assume that all the previously defined mappings $f_{i, n}$ are equal to a unique linear mapping, given by:

$p_{i, n}=\left(\alpha \cdot \Gamma_{i, n}: \alpha \in[0,1]\right) \quad \forall(i, n) \in \mathcal{J}_{n} \times \mathcal{N}$,

where $\alpha$ is included to model a broad range of investment behaviours, e. g. a small value implies a increased tendency to invest. Then, the random variable $\beta_{i, n}$, that controls the decision of investing or not in a DER installation of size $A_{G_{i, n}}^{*}$, is drawn from the Bernoulli distribution $B(1$, $\left.p_{i, n}\right)$ : 
$\beta_{i, n} \sim B\left(1, p_{i, n}\right) \quad \forall(i, n) \in \mathcal{J}_{n} \times \mathcal{N}$.

Finally, the decision for every potential prosumer is given by:

$\theta_{i, n}=1-\beta_{i, n} \quad \forall(i, n) \in \mathcal{J}_{n} \times \mathcal{N}$,

with $\theta_{i, n} \in\{0,1\}$ by definition of the Bernoulli distribution. If $\theta_{i, n}=1$, a DER installation of size $A_{G_{i, n}}^{*}$ is deployed by the agent $i$. This agent is then removed from the set of users $\mathcal{J}_{n}$. In this way, when a DER installation of size $A_{G_{i, n}}^{*}$ is deployed, the user $i$ is prevented from investing in the future. If $\theta_{i, n}=0$, the DER installation is not deployed, and another opportunity will be given to user $i$ at the following step $n+1$. The set $\mathcal{J}_{n+1}$ can thus be computed as follows:

$\mathcal{J}_{n+1}=\mathcal{J}_{n} \backslash\left\{i: \theta_{i, n}=1\right\}$.

Modelling the investment decision-making process in such fashion ensures the deployment of some DER units even when the viability of the DER installations lie at the economically feasible limit (for instance when the technology costs are high or the retail price of electricity is low), representing the behaviour of those users who are eager to invest. Likewise, this investment decision-making mechanism will prevent some agents from investing even under favourable conditions, representing those agents more reluctant to invest. Also, slightly randomising the decision process using a Bernoulli distribution allows to aggregate the effect of variables that influence the decision-making process but that are not explicitly modelled in this article, such as the access to capital for investing in DER, or the interest in renewable energy.

\subsection{Distribution system operator's remuneration mechanism}

The DSO distributes electricity to the users of the distribution network, charging a distribution fee for the service. This fee must be sufficiently large so as to collect the revenue that allows the DSO to break-even financially. Hence, assigning an adequate level of a distribution fee is a delicate process. An under-estimated fee may lead to insufficient remuneration, creating an economic imbalance that must eventually be socialised via higher rates. On the other hand, an inflated tariff may place excessive economic strain on users. Both deviations from the optimum are symptoms of an inefficient DSO remuneration strategy. To model the interactions of the DSO, we represent its remuneration mechanism, which includes the adjustment of the distribution fee when needed. Note that the tariff design cannot be modified by the DSO since it is controlled by the incumbent regulatory authority.

The remuneration mechanism computes the distribution fee by comparing the costs $\left(\Theta_{n}\right)$ and the revenue $\left(R_{n}\right)$ of the DSO in the previous tariff period and computing its difference $\Delta_{n}=\Theta_{n}-R_{n}$. If $\Delta_{n}=0$, it means that the distribution tariff level is adequate. However, if $\Delta_{n}>0$ or $\Delta_{n}<0$, it indicates an under- or over-estimation of the distribution fee, respectively. It is important to note that the applied fee is always an estimation of the real one, based on forecasts of consumption. In our work, we assume that the forecast used by the DSO is a continuation of the last observed state of the system. Furthermore, we assume that at the initial state, the system is economically balanced, i.e. $\Delta_{-1}=0$ and therefore $\Theta_{-1}=R_{-1}$. Hence, the initial costs of the system can be calculated by determining the initial revenue. The general expression to compute the DSO revenue is:

$$
\begin{aligned}
& {\left[\Pi_{n}^{(v o l)} \cdot\left(\Omega+\Xi_{n}\right)\right] } \\
R_{n}=+ & {\left[\Pi_{n}^{(c a p)} \cdot \sum_{i=1}^{\left(I+I_{0}\right)} \gamma_{i, n}\right] \quad \forall n \in \mathcal{N}, } \\
& +\left[\Pi_{n}^{(f i x)} \cdot\left(I+I_{0}\right)\right]
\end{aligned}
$$

where $\Pi_{n}^{(v o l)}, \Pi_{n}^{(c a p)}$, and $\Pi_{n}^{(f i x)}$ represent the volumetric, capacity, and fixed fees, respectively, at the $n^{\text {th }}$ time-step. $I_{0}$ stands for the number of consumers who make up the residual demand (i.e. non-prosumers). $\gamma_{i, n}$ represents the optimised peak demand of the $i^{\text {th }}$ user, output of the MILP. $\Omega$ represents the residual demand of the system, which is an input of the simulation and is held constant throughout the entire simulation process. Finally, $\Xi_{n}$ represents the aggregated consumption of the agents in $\mathcal{I}$, computed as:

$\Xi_{n}=\sum_{i=1}^{I} \rho_{i, n}^{(-)} \quad \forall n \in \mathcal{N}$,

where $\rho_{i, n}^{(-)}$represents the optimised imports of the $i^{\text {th }}$ potential or actual prosumer at the $n^{\text {th }}$ time-step, which is an output of the MILP.

To begin the simulation we need the initial costs $\left(\Theta_{-1}\right)$, these are, as previously explained, equal to the initial revenue $\left(R_{-1}\right)$. The latter can be easily computed by means of Eq. (30), since the demand profiles of the potential prosumers and the residual demand are known. Once the initial revenue (and therefore the initial costs) of the DSO are computed, the remuneration mechanism can distribute them across the different types of fees: volumetric, capacity, or fixed, thus obtaining three different fees which are applied to the final customers' electricity bills (note that ToU fees are a particular case of volumetric fees). The same distribution mechanism is used for computing the initial fees and to update them in subsequent time-steps of our discrete-time dynamical system. Such a computation is given by the following expressions:

$\Pi_{n+1}^{(v o l)}=\left[\frac{\Theta_{n}+\Delta_{n}}{\Omega+\Xi_{n}}\right] \cdot \mu_{1} \quad \forall n \in \mathcal{N}$,

$\Pi_{n+1}^{(c a p)}=\left[\frac{\Theta_{n}+\Delta_{n}}{\sum_{i=0}^{\left(I+I_{0}\right)} \gamma_{i, n}}\right] \cdot \mu_{2} \quad \forall n \in \mathcal{N}$,

$\Pi_{n+1}^{(f i x)}=\left[\frac{\Theta_{n}+\Delta_{n}}{I+I_{0}}\right] \cdot \mu_{3} \quad \forall n \in \mathcal{N}$

In these equations, $\mu_{1}, \mu_{2}$, and $\mu_{3}$ represent the share of the volumetric, capacity, and fixed fee, respectively, imposed by the DSO remuneration strategy, and thereby by the regulatory framework set by the regulator. These shares comply with $\sum_{j=1}^{3} \mu_{j}=1$.

To compute the fees for time-step $n=0$, we know $\Theta_{-1}$ as it equals the revenue at this time-step. Furthermore, we know that $\Delta_{-1}=0$. The rest of the elements in Eqs. (32)-(34) are given by the profiles of the users, which are known. Once the simulation starts, at every time-step $n$, some potential prosumers may turn into actual prosumers, impacting the revenue of the DSO and, in particular, $\Xi_{n}$ and $\gamma_{i, n}$. The DSO, in turn, reacts by updating the different components of the distribution tariff. Finally, since we work under the assumption that the DSO uses its last observed state of the system as forecast for the following tariff period, the costs at a given period will be the same as the revenue at the previous one $\Theta_{n}=R_{n-1}$.

\subsection{User's electricity bill}

The electricity bills of the distribution network's final customers depend on their imports and their exports (if any) of electricity. In this paper, we assume a full roll out of smart meters, therefore these two electricity flows are registered independently by the metering device, and have two different price signals associated.

Imports of electricity: This is the overall price of electricity the final customers (consumers and potential and actual prosumers) pay to use the network and consume electricity from it. This price includes commodity, transmission, distribution, and other levies. In this work we are interested in the distribution part, therefore, all the other elements making up the electricity price are grouped in one element, $\Pi^{(o t)}$, 
introduced in Eq. (6) and set in $€ / \mathrm{kWh}$. As for the distribution fee, the smart meters allow us to split the distribution component of the electricity bill into its constituents: $\Pi^{(v o l)}, \Pi^{(c a p)}$, and $\Pi^{(f i x)}$, as in (5). The contribution of each element is given by $\mu_{j}$ (see Eqs. (32)-(34)) and depends on the DSO remuneration mechanism.

Exports of electricity: This is the selling price of the actual prosumers when exporting electricity to the grid. It is introduced by $\Pi^{(s p)}$ in Eq. (19) and set in $€ / \mathrm{kWh}$.

\section{Test case: simulator demonstration}

To test and illustrate the proposed simulation-based approach, this section presents an extensive range of tests showcasing the potential of the presented methodology to flexibly simulate a wide range of scenarios. To create these scenarios, we need: (i) a set of users, and (ii) a set of rules representing a regulatory framework (designing the DSO remuneration strategy). Then, by using the same set of users for different remuneration strategies, we can analyse the impact of the latter on different features inherent to distribution networks, notably the distribution network prices and the level of penetration of distributed generation in the distribution network.

\section{Set of users:}

Users are characterised by individual demand and production profiles. A bottom-up approach, the CREST model [32], was used to generate demand profiles. Using the CREST model we produced a range of daily profiles representing weekends and weekdays and then, by means of a randomisation process, different demand profiles spanning one year and with a resolution of one hour, were generated. As for the production profiles, they were generated with the same time span and resolution (one year and one hour, respectively), representing the potential for PV generation of prosumers. To do so, the Python library PVLIB [33] was used. The profiles thus produced are based on solar radiation historical data, obtained through typical meteorological years (tmy), which were downloaded from the Joint Research Centre of the European Commission. ${ }^{2}$ From a range of different tmy, and making variations on the tilt and orientation of the PV panels (parameters of PVLIB), different profiles were generated. These profiles represent the load factor, i.e. percentage of the total installed capacity that is produced at each time-step.

In total, 1,000 demand and production profiles were generated, to represent 1,000 potential prosumers. In addition to them, 5,000 consumers were created for whom only the aggregated yearly demand is needed as they make up what we call residual demand of the system. Both groups of customers (consumers and prosumers) have been created according to the Belgian reality, that is, the profiles are consistent with electricity consumption and solar radiation in Belgium. The proportion prosumers/consumers is selected so as to reflect the real-life situation in Belgium, as described in [34].

Set of rules of a regulatory framework

Two groups of scenarios are proposed:

- Simulation-based approach capabilities: First we generate several scenarios showcasing the capabilities of the proposed simulationbased approach to compute a prediction of the evolution of distribution network features (distribution prices and penetration of DER). These scenarios represent various DSO remuneration strategies.

- Sensitivity analyses: Then, the sensitivity of our approach to several parameters is tested, reporting on the impacts these parameters have on the simulation-based approach capabilities to predict the distribution network development.

\footnotetext{
2 Joint Research Centre photovoltaic geographical information system https://re.jrc.ec.europa.eu/pvg_tools/en/tools.html\#TMY.
}

\subsection{Simulation-based approach capabilities}

In this part of the simulation results, we test seven scenarios mimicking different initial conditions set by the regulator. Accordingly, we can introduce different values of $\mu_{j}$ for each scenario. These values will impact on the evolution of the different elements of the distribution tariff, as described by Eqs. (32)-(34). In these equations, all the variables are known. Therefore, to start the simulations we only need an initial state, i.e. the initial costs of the system (by assumption equal to the initial revenue $\Theta_{-1}=R_{-1}$ ). To compute the initial revenue, in this example we use the current situation in Belgium, where the distribution fee is based on a volumetric tariff which, on average, amounts to 0.08 $€ / \mathrm{kWh}$ (i.e. $\Pi_{-1}^{(v o l)}=0.08, \Pi_{-1}^{(c a p)}=0, \Pi_{-1}^{(f i x)}=0$ ) and determine $R_{-1}$ as expressed in Eq. (30). Since this initial revenue must be the same regardless of the scenario we want to test, we can break it down for different initial states representing different distributions of volume, capacity, and fixed fees (i.e. different scenarios), using Eqs. (32)-(34). Using this procedure, we have built seven scenarios, showcasing a range of different possible tariff designs. Along with these, one additional scenario has been created to test the impact of ToU distribution tariffs based on volumetric fees. All these scenarios are listed in Table 1.

Finally, Table 2 lists the rest of the inputs used to run the scenarios. To assess each scenario, we use three metrics: (i) the penetration of actual prosumers relative to the maximum potential; (ii) the evolution of the electricity costs for consumers and prosumers; and (iii) the actual deployed PV and battery capacities (in kWp and $\mathrm{kWh}$ respectively).

\subsubsection{Results}

To quantitatively show the evolution of the penetration of actual prosumers over time, Fig. 1 presents the percentage of actual prosumers with respect to the maximum potential, for each time-step of the

Table 1

Construction of the different scenarios.

\begin{tabular}{|c|c|c|c|c|}
\hline Scenario & Description & $\mu_{1}$ & $\mu_{2}$ & $\mu_{3}$ \\
\hline VOL & $\begin{array}{l}\text { Based on fully } \\
\text { volumetric distribution } \\
\text { fees }\end{array}$ & 1 & 0 & 0 \\
\hline CAP & $\begin{array}{l}\text { Based on fully capacity } \\
\text { distribution fees }\end{array}$ & 0 & 1 & 0 \\
\hline FIX & $\begin{array}{l}\text { Based on fully fixed } \\
\text { fees, per connection } \\
\text { point }\end{array}$ & 0 & 0 & 1 \\
\hline VOL_CAP & $\begin{array}{l}\text { Based } 50 \% \text { on volume } \\
\text { and } 50 \% \text { on capacity } \\
\text { fees }\end{array}$ & $1 / 2$ & $1 / 2$ & 0 \\
\hline VOL_FIX & $\begin{array}{l}\text { Based } 50 \% \text { on volume } \\
\text { and } 50 \% \text { on fixed fees }\end{array}$ & $1 / 2$ & 0 & $1 / 2$ \\
\hline CAP_FIX & $\begin{array}{l}\text { Based } 50 \% \text { on capacity } \\
\text { and } 50 \% \text { on fixed fees }\end{array}$ & 0 & $1 / 2$ & $1 / 2$ \\
\hline EVEN & $\begin{array}{l}\text { Based on a even } \\
\text { distribution of the } \\
\text { weights }\end{array}$ & $1 / 3$ & $1 / 3$ & $1 / 3$ \\
\hline TOU & Time-of-use tariff* & 1 & 0 & 0 \\
\hline
\end{tabular}

* The ToU distribution tariff is created by using a fully volumetric fee such as VOL, where different levels of the fee are applied depending on the time of the day. In our particular example, three different levels are applied corresponding to peak rates, off-peak rates, and shoulder rates.

dynamical system. Furthermore, to show the evolution of the distribution tariff, driven by Eqs. (32)-(34), we compute the total electricity costs for consumers, which depict the same evolution as only the distribution component of the overall retail electricity tariff can change 
Table 2

General inputs of the multi-agent model.

\begin{tabular}{ccc}
\hline Parameter & Value & Units \\
\hline$P^{(p v) *}$ & 1,200 & {$[€ / \mathrm{kWp}]$} \\
$Q^{(p v) *}$ & 500 & {$[€]$} \\
$P^{(b a t) *}$ & 200 & {$[€ / \mathrm{kWh}]$} \\
$Q^{(b a t) *}$ & 200 & {$[€]$} \\
$\Pi_{n}^{(o t)}$ & 0.132 & {$[€ / \mathrm{kWh}]$} \\
$\eta^{(-)}$ & 0.95 & {$[\%]$} \\
$\eta^{(+)}$ & 0.95 & {$[\%]$} \\
$F^{(-)}$ & 2.5 & {$[-]$} \\
$F^{(+)}$ & 4 & {$[-]$} \\
$B$ & 8 & {$[\mathrm{years}]$} \\
$\bar{p}$ & 10 & {$[\mathrm{kWp}]$} \\
$\bar{b}$ & 30 & {$[\mathrm{kWh}]$} \\
$\alpha$ & 1 & {$[-]$} \\
$Y$ & 20 & {$[\mathrm{years}]$} \\
$r$ & 0.02 & {$[\%]$} \\
$\Omega$ & 1,000 & {$[\mathrm{kWh}]$} \\
$I$ & $85 \%$ of total load & {$[\#]$} \\
\hline
\end{tabular}

* Prices at time $n=0$, they linearly decrease over time by $5 \%$ each tariff period.

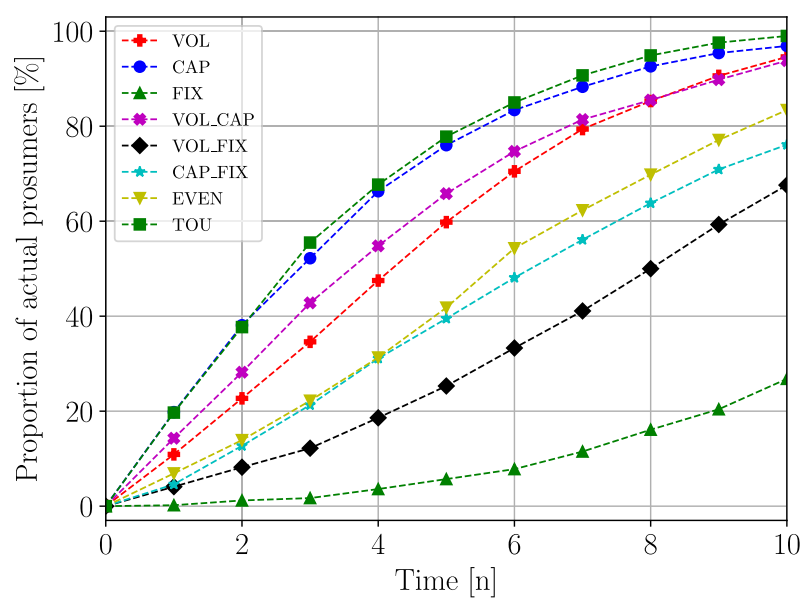

Fig. 1. Penetration of DER in the distribution network as a proportion of the total potential penetration over time.

over time. ${ }^{3}$ We compute these costs at each time-step and normalise them by the initial costs $t=0$, displaying the evolution in Fig. 2 .

On the one hand these plots show the effectiveness of each scenario to stimulate the adoption of PV and batteries (i.e. of prosumers), and on the other hand the repercussions of such a deployment in terms of electricity costs for the regular consumers of the distribution network. In these examples, all the scenarios with the exception of the one based on only fixed fees (FIX), lead to increased electricity costs. However, the information in these plots is incomplete, since they do not provide any details on the actual amount of PV and batteries deployed by prosumers. Fig. 3 shows the total accumulated installed capacity of PV and batteries for each scenario. This information adds to that previously provided by including details of the composition of the prosumers' installations.

\footnotetext{
${ }^{3}$ Note that for this calculation only consumers are used and not prosumers. The reason for this is that the total electricity costs of prosumers depend on their DER installations as well as on the distribution tariff, and consequently the evolution described by these total costs is not equal to the one described by the distribution tariff alone. Therefore, as our only interest is to show the evolution of the distribution tariff, prosumers can be left out from this computation.
}

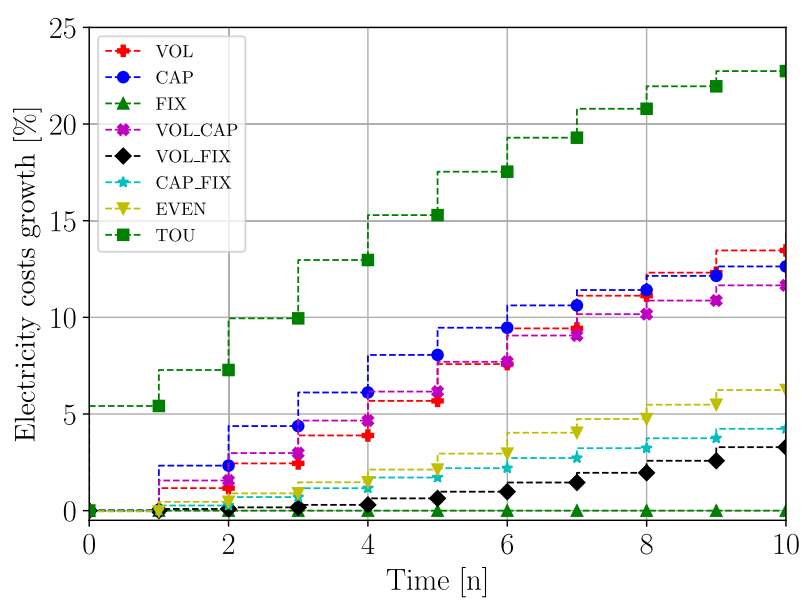

Fig. 2. Growth of the overall electricity cost for consumers over time.

Finally, Table 3 shows the annual electricity costs for an average consumer and an average prosumer at the end of the simulated period (i. e. at time-step 10). This provides the actual value in $€$ consumers and prosumers pay to cover their electricity needs for each scenario.

We can extract a few general remarks from Figs. 1-3, and from Table 3.

- Tariff structures prominently based on volumetric fees induce a very significant deployment of PV panels and batteries (mainly the former) as well as rapid transition from potential to actual prosumer. This deployment is followed by a large growth of the overall electricity costs for consumers. Moreover, these tariffs lead to substantial exports from prosumers' DER installations to the distribution network, owing to larger PV capacities. In addition, these tariff structures lead to substantial inequalities in the electricity costs, in particular when no other component is added to the tariff (i.e., fully volumetric structures such as VOL and TOU) - in these cases the economic burden of maintaining the DSO is mostly carried by consumers.

- When the tariff design is weighted toward capacity fees, the deployment of PV panels and batteries is also spurred, although to a lesser extent and inclines the balance toward more batteries this time. However, the induced increase in electricity costs is larger than in the previous case. The bias of these scenarios toward using batteries is explained by the ability of actual prosumers to shave their peaks ( $\gamma$ in Eq. (5)) thus paying less in capacity fees. This is consistent with the findings in [35]. Moreover, tariffs based on these fees tend to import more electricity than export it - this electricity is stored in the larger batteries to shave the peak demand. Regarding the cost distribution shown in Table 3, these types of tariff result in highly unequal distributions, similar to those observed with volumetric fees, where the financial burden of the DSO is born by consumers.

- Adding a fixed term helps reduce the impact on the electricity costs for consumers in either volumetric or capacity fees. However, using purely fixed fees does not seem to promote the deployment of PV panels and batteries, in particular the latter. Balancing several part tariffs results in a trade-off that must be carefully studied as suggested in [14], falling outwith the scope of our work.

- Using ToU tariffs creates the more extreme outcome - the quickest transition from potential to actual prosumers among all assessed scenarios is only followed by the largest increase in electricity costs for consumers. The incentive to install PV panels is the second largest (after VOL), whereas the incentive to install batteries is the largest one. These results are explained by the possibility of actual prosumers benefiting from both PV panels to limit their exposure to the volumetric fees and batteries to shift load from peak and off-peak to 


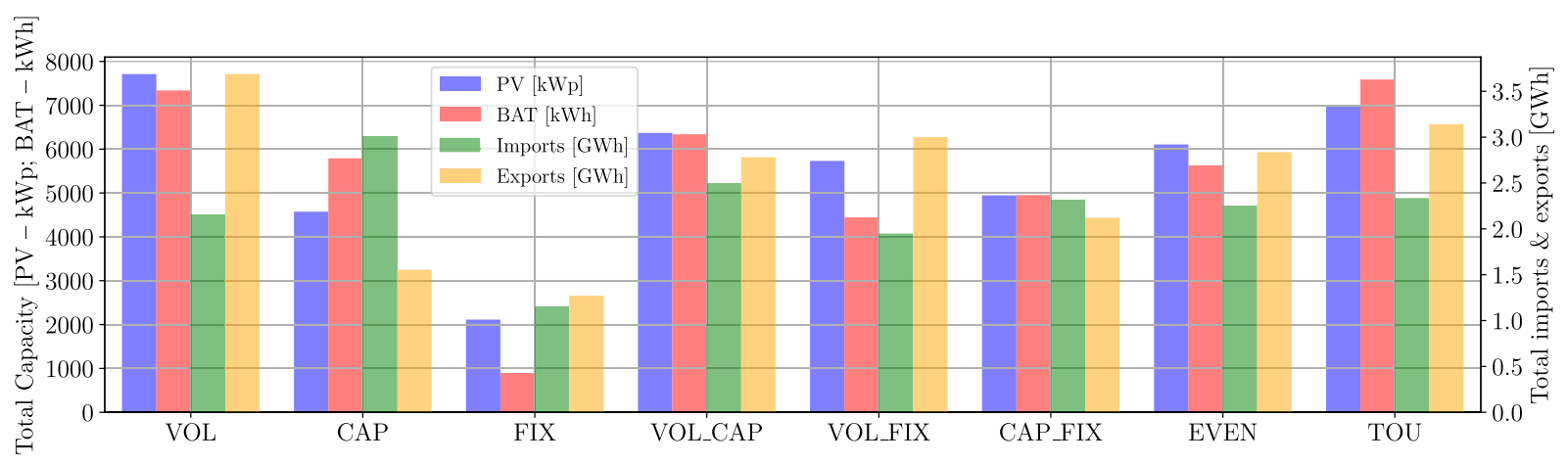

Fig. 3. Total capacity of installed PV capacity (blue), total capacity of installed battery (red), total imports from the distribution network (green), and total exports to the distribution network (yellow) at the end of the simulation period. (For interpretation of the references to colour in this figure legend, the reader is referred to the web version of this article.)

Table 3

Annual electricity costs for an average consumer and an average actual prosumer at the end of the simulated period.

\begin{tabular}{lll}
\hline Scenario & Annual consumer costs $[€]$ & Annual prosumer costs $[€]$ \\
\hline VOL & $1,514.52$ & $1,063.21$ \\
CAP & $1,491.59$ & $1,008.10$ \\
FIX & $1,317.79$ & $1,235.72$ \\
VOL_CAP & $1,487.72$ & $1,043.81$ \\
VOL_FIX & $1,378.31$ & $1,149.53$ \\
CAP_FIX & $1,384.60$ & $1,139.22$ \\
EVEN & $1,415.09$ & $1,112.93$ \\
TOU & $1,624.50$ & $1,085.86$ \\
\hline
\end{tabular}

shoulder hours $\left(\rho_{t}^{(-)}\right.$in Eq. (8)). In a similar way as with volumetric fees, ToU fees lead to more exports than imports. However, in this case, the spread between both is smaller, since the electricity surplus with ToU tariffs can be stored in the batteries to shift demand.

\subsubsection{Discussion}

These analyses show that different initial conditions, notably including various tariff structures, induce vastly different outputs that can be quantitatively assessed. The presented simulation environment can be used to discriminate between the possible outcomes of employing distinct tariff structures. It may therefore be valuable for assessing a distribution tariff structure before putting it in force.

Extracting meaningful conclusions with this tool necessitates a previous tuning phase where the various parameters of the simulationbased approach (e.g. $\alpha$, prices, bounds $\bar{p}$ and $\bar{b}$ ) must be adapted to the particular context that one wants to simulate. In this regard, in [34] this simulation-based approach is employed to simulate a distribution network with the characteristics inherent to the Walloon region of Belgium, providing policy recommendations for this particular case.

Although the example provided in this section does not correspond to any particular context (i.e., no previous tuning phase has been performed to adapt the simulation-based approach to any particular case), there are a few general observations that can be drawn. In sensitivity, these observations are based on the principles for distribution tariff design, as presented in $[8,9]$.

- Distribution tariff designs based on volumetric fees (totally or partially) promote the largest adoptions of PV panels and batteries; however, they lead to the largest inequalities between consumers and prosumers. On the one hand, their implementation is straight forward, complying with the principles of transparency, simplicity, and predictability. On the other hand, they distort the decisions concerning the use of the network, they are not cost reflective (in fact prosumers, who use the network more, end up paying significantly less than consumers), resulting in discrimination among the users where not all of them pay the same for the same service, leading to cross-subsidies from consumers to prosumers.

- Capacity charges lead to high battery deployment and relatively high PV panel adoption. However, as with volumetric charges, the economic inequalities between consumers and prosumers are substantial. In terms of the tariff design principles, capacity charges are not as predictable, transparent and simple as volumetric ones, and moreover they induce distortion and discrimination among the network users. Finally their cost-reflectivity in already developed distribution networks is questionable, as typically network costs are sunk.

- Fixed fees comply with most of the principles of tariff design (nondistorting, non-discriminatory, transparent, predictable and simple). However, they do not promote the adoption of DER installations, which has been a high-level goal of all energy policies over the last few years (see for instance [36]).

- Applying ToU charges on top of volumetric ones results in the largest battery deployment and the second-largest PV panel deployment leading to the highest cost different between consumers and prosumers where the former pay substantially more than the latter for their network use (cross-subsidies). This type of charges is transparent, predictable and relatively simple, although, as volumetric ones, they are not cost-reflective and they may distort and discriminate among users.

- Tariffs based on a mix between different types of charge result in a trade-off between promoting PV panels and battery adoption, distributing the costs among the users in a more equal fashion, and complying with some of the principles of distribution tariff design.

These remarks confirm that selecting the tariff design is not a trivial process, where no perfect tariff exists. In general, applying fully volumetric, capacity, or fixed fees does not seem to yield the most adequate results where either DERs are not promoted, or they are promoted but the costs for it are mostly born by consumers instead of prosumers (who generate most of them), leading to cross-subsidies. A solution could be to resort to designs where all these components are present. In these cases, a middle-ground target can be achieved, promoting some DER adoption whilst maintaining a relative level playing field for consumers and prosumers. Overall, promoting DER has a cost associated, and it is the 
decision of regulators and policy makers to choose how to cover it.

\subsection{Sensitivity analyses}

In this section, the sensitivity of the proposed simulation-based approach to several parameters is tested. In particular, we perform sensitivity analyses on the $\alpha$ parameter, the selling price of electricity for prosumers (sp), and the prices of PV panels and batteries (tp). For these analyses we use the same basic data listed in Table 2, only modifying the parameter we wish to study.

\subsubsection{Sensitivity to $\alpha$}

The first of the analyses presented in this section corresponds to the sensitivity to the $\alpha$ parameter. As explained in Section 4.5, this parameter controls the speed at which the DERs are deployed by potential prosumers. It works by biasing the $p$ parameter of a Bernoulli random variable (i.e. the probability of drawing a 0 or a 1 ). This $p$ parameter is computed as the difference between the LVOE of a DER installation and the electricity costs the potential prosumer would otherwise face without the DER installation. We further develop this definition introducing the $\alpha$ parameter which we multiply by the cost difference (see Eq. (26)). Since the investment decision is inverted (see Eq. (28)), a low value of $\alpha$ fosters the deployment of DER whilst a high value limits it.

As we can observe in Figs. 4 and 5 and Table 4, the lower the $\alpha$, the greater the deployment of DER. This greater DER penetration, in turn, results in a higher increase of the overall electricity cost of traditional consumers. When looking at the total PV capacity and battery capacity deployed, it can be noted that for low values of $\alpha$ the resulting total capacity is lower than for values close to 1 . This is explained by the fact that, since the DER installations need to be more profitable when $\alpha=1$ than when $\alpha=0.6$ to be deployed (i.e to draw a 0 in the Bernoulli random variable), only large and profitable installations will be deployed. This behaviour results apparent when $\alpha>1$. However, since in those cases the simulator does not reach $100 \%$ of DER deployment, the total DER capacity at the end of the simulation horizon is lower than for $\alpha=1$. A longer simulation horizon will prove those scenarios to

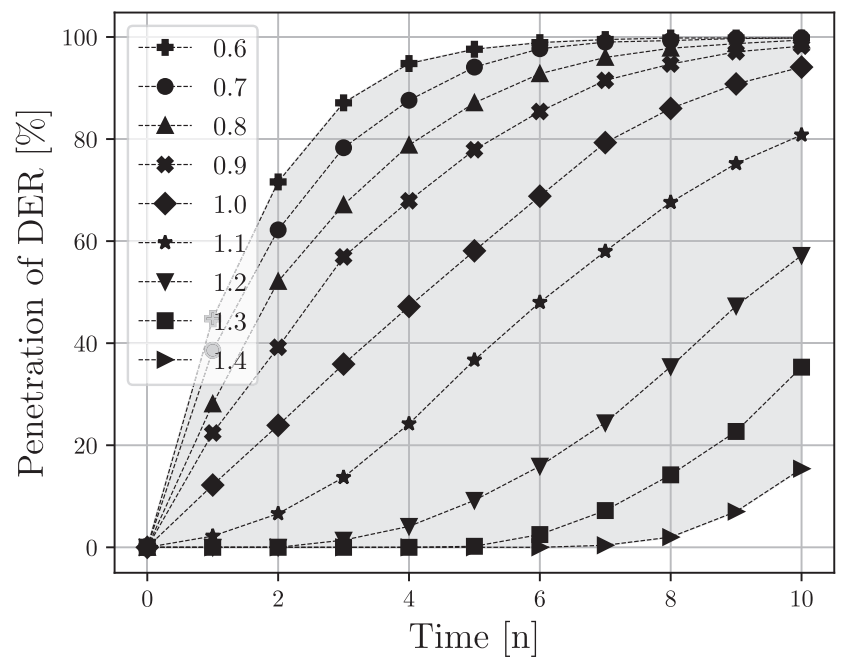

Fig. 4. Sensitivity of DER deployment to $\alpha$.

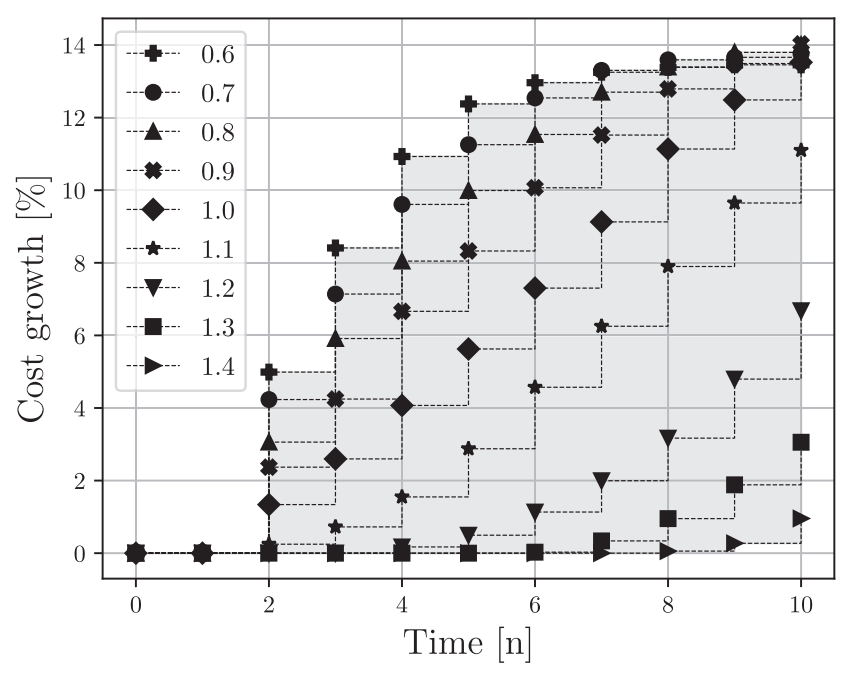

Fig. 5. Sensitivity of electricity costs for consumers to $\alpha$.

present the largest total deployment of PV panels and batteries. This parameter presents an enormous variability in the outcome. This is why, before making use of the proposed simulation-based approach, it is key to tune this parameter to adapt it to the conditions of the distribution network it aims to simulate, as done in [34].

\subsubsection{Sensitivity to the selling price}

The second analysis tests the sensitivity of the model to the selling price of electricity of prosumers. These users primarily use their local electricity production to meet their demand, however, when there is more production than demand, they can sell this surplus to the distribution network. Modifying the selling price has therefore the potential to affect the behaviour of those users as the value associated to their electricity exports changes.

From Fig. 6 we can observe that higher selling prices lead to greater DER adoption and, in turn, to an overall increase in electricity costs, as

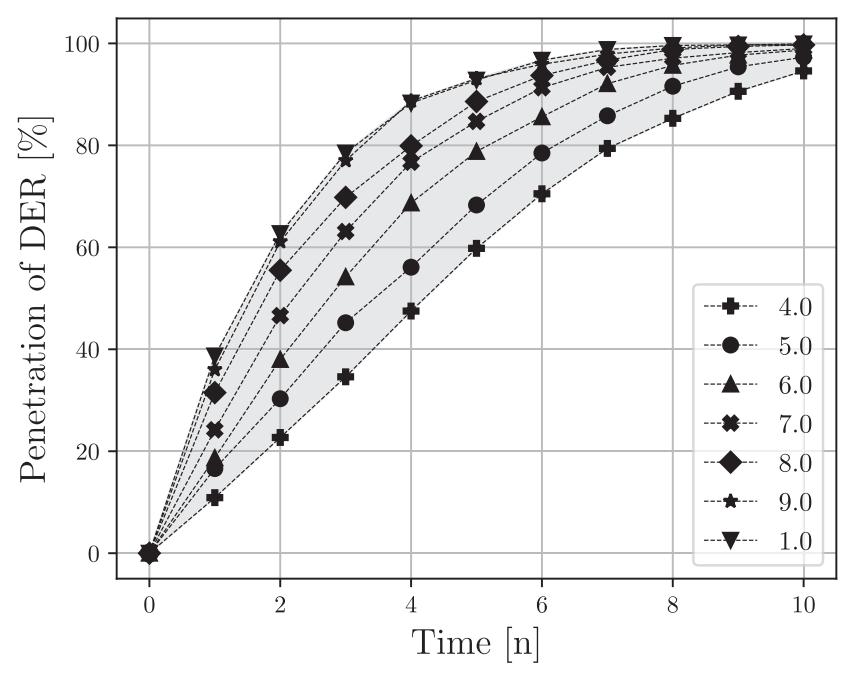

Fig. 6. Sensitivity of DER deployment to the selling price (in c€).

Table 4

Sensitivity of PV- and battery-installed capacity to $\alpha$.

\begin{tabular}{|c|c|c|c|c|c|c|c|c|c|}
\hline & $\alpha=0.6$ & $\alpha=0.7$ & $\alpha=0.8$ & $\alpha=0.9$ & $\alpha=1.0$ & $\alpha=1.1$ & $\alpha=1.2$ & $\alpha=1.3$ & $\alpha=1.4$ \\
\hline Total PV capacity [kWp] & $6,071.4$ & $6,374.6$ & $6,738.7$ & $7,124.9$ & $7,673.1$ & $7,456.1$ & $6,506.9$ & $4,688.2$ & $2,460.0$ \\
\hline Total battery $[\mathrm{kWh}]$ & $6,379.8$ & $6,571.9$ & $6,828.7$ & $7,009.1$ & $7,295.6$ & $6,833.6$ & $5,713.4$ & $4,061.8$ & $2,161.2$ \\
\hline
\end{tabular}




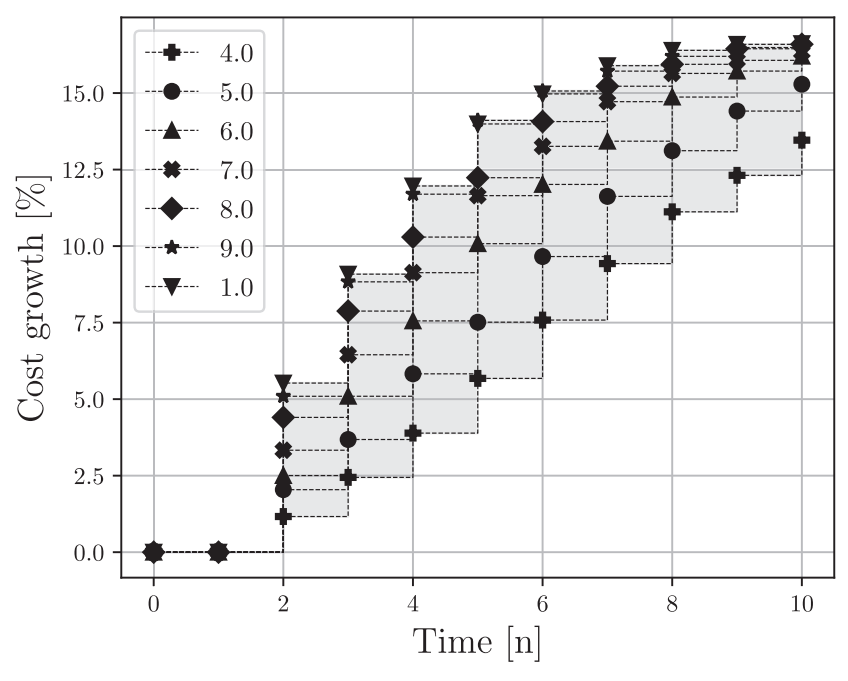

Fig. 7. Sensitivity of electricity costs for consumers to the selling price (in $c €$ ).

shown in Fig. 7. From the total deployed capacity of PV and batteries (Table 5), it can be deduced that the greater the selling price, the larger the PV installation. This relation, nonetheless, is the opposite in the case of batteries, where a higher selling price leads to lower battery adoption. These effects are a result of the business model of these two behind-themeter devices. A greater PV capacity results in a larger production surplus that can be sold to the network and, therefore, a higher selling price will spur larger PV installations with substantial production surpluses. Larger PV installations will in turn require fewer batteries to operate, since they will produce sufficient electricity to cover the prosumers' demand, even at times where there is a limited solar availability. Even though the selling price clearly imposes some changes in the adoption rate of PV and batteries, these changes are less significant than in our first analysis. The selection of this parameter is easier than the other two ( $\alpha$ and technology price) for it should reflect the regulation in place.

\subsubsection{Sensitivity to the technology price}

The last of the introduced analyses deals with the sensitivity to the technology price. To carry out this assessment, as the starting point we take the technology costs (PV and battery) listed in Table 2. We then multiply them by a factor to increase or decrease the initial technology costs, analysing the sensitivity to different factors (costs), as shown in Table 6. Note that these are the initial technology costs, which then decrease by $5 \%$ every year.
In Figs. 8 and 9, a linear relation can be seen between technology price and adoption rate of PV and batteries. Unsurprisingly, the higher the price the lower the penetration of actual prosumers and, as such, the lower the impact on overall electricity costs. On the other hand, the lower the technology price, the faster and larger the deployment of DER installations. As expected, this parameter has a strong influence on the shape of the trend curves - it is therefore crucial to find the right level of technology prices.

\section{Conclusion}

This paper formalises and builds a framework based on a simulationbased approach to assess the impact of a wide range of DSO remuneration strategies on the economic sustainability of the distribution network. The potential of this simulation-based approach lies in its ability to accurately discriminate between the possible outcomes of employing distinct remuneration strategies in order to provide sound arguments that underpin the selection of one of them. It therefore serves as guidance for policy makers and regulators to build new remuneration strategies for DSOs, aiming to achieve certain specific objectives (e.g. promote the adoption of renewable distributed generation). By means of this simulation-based approach they can compare the strengths and drawbacks of distinct options before applying them in real life. The proposed simulation environment contributes to the existing literature by:

- Providing the mathematical formalisation of a simulation-based approach based on a dynamical system and on an optimisation framework that progressively deploys DER installations over time and, as a result, adapt the distribution network charges to make sure any imbalance of the DSO is corrected.

- Encapsulating all the most commonly used DSO remuneration strategies -(i) volumetric fees based on energy consumed, (ii) capacity fees based on power withdrawn, (iii) fixed fees based on the availability of a connection point, and (iv) time-of-use fees that depend on the time of energy consumption- in the developed mathematical formalisation of the simulation-based approach.

- Providing a computational tool encoding such a mathematical formalisation to help policy makers and regulators decide which DSO remuneration strategy to employ according to a specific target. ${ }^{4}$

The simulation-based approach presented in this paper is formulated to be sufficiently generic so that it can adapt to any context (i.e. any distribution network) with ease by tuning certain parameters as, for instance, the Bernoulli bias $\alpha$, or the technology costs. This means that before being used, this tool must be tuned so as to match the distribution

Table 5

Sensitivity of PV- and battery-installed capacity to the selling price (in c€).

\begin{tabular}{|c|c|c|c|c|c|c|c|}
\hline & $\mathrm{sp}=4$ & $\mathrm{sp}=5$ & $\mathrm{sp}=6$ & $\mathrm{sp}=7$ & $\mathrm{sp}=8$ & $\mathrm{sp}=9$ & $\mathrm{sp}=10$ \\
\hline Total PV capacity [kWp] & $7,712.4$ & $8,961.0$ & $9,748.5$ & $9,928.5$ & $9,970.0$ & $9,970.0$ & $9,970.0$ \\
\hline Total battery $[\mathrm{kWh}]$ & $7,339.0$ & $7,484.4$ & $7,442.9$ & $7,384.4$ & $7,293.4$ & $7,217.9$ & $7,204.4$ \\
\hline
\end{tabular}

Table 6

Sensitivity of PV- and battery-installed capacity to the technology price. Note that the shown percentages are relative to the prices used for the first simulation.

\begin{tabular}{|c|c|c|c|c|c|c|c|}
\hline & $\mathrm{tp}=70 \%$ & $\mathrm{tp}=80 \%$ & $\mathrm{tp}=90 \%$ & $\mathrm{tp}=100 \%$ & $\mathrm{tp}=110 \%$ & $\mathrm{tp}=120 \%$ & $\mathrm{tp}=130 \%$ \\
\hline Total PV capacity [kWp] & $9,942.0$ & $9,436.6$ & $8,502.8$ & $7,541.1$ & $6,584.7$ & $5,751.9$ & $4,225.1$ \\
\hline Total battery $[\mathrm{kWh}]$ & $8,857.0$ & $8,383.7$ & $7,847.4$ & $7,187.2$ & $6,409.5$ & $5,417.4$ & $3,925.5$ \\
\hline
\end{tabular}

\footnotetext{
${ }^{4}$ A computational tool encoding the proposed simulation based approach can be found in https://github.com/manueldevillena/tariff_simulator.
} 


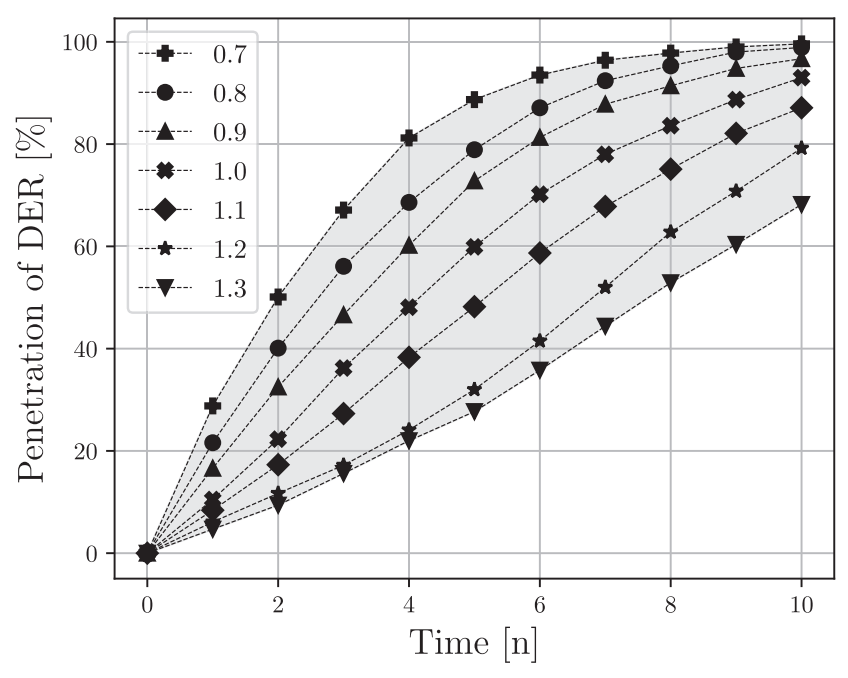

Fig. 8. Sensitivity of DER deployment to the technology price (the numbers indicate the value by which the initial PV and battery prices from Table 2 are multiplied).

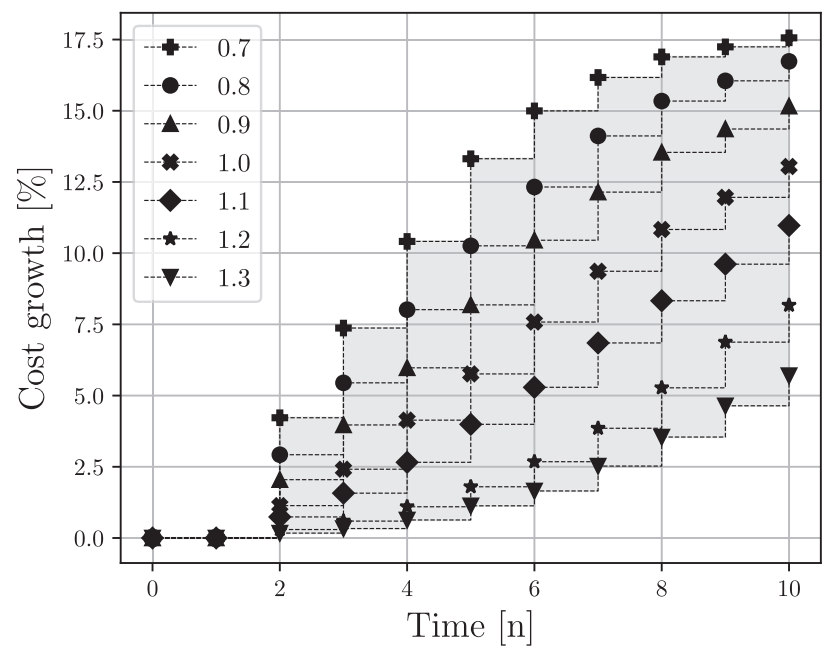

Fig. 9. Sensitivity of electricity costs for consumers to the technology price the numbers indicate the value by which the initial PV and battery prices from Table 2 are multiplied).

network where the experiments are to be run.

The presented approach has been extensively tested with a case study featuring eight different scenarios which illustrate the various options of the proposed simulation environment to guide future developments in distribution network tariff structures. This test case shows how prosumers' choices vary from one remuneration strategy to another, suggesting that it is valuable to check by simulation that a remuneration strategy yields the desired outcome. Although this test case does not intend to offer insights regarding any particular distribution network (i. e. the parameters are set by default), it does provide some guidance on how to design the remuneration strategy of a DSO:

- Strategies based on volumetric fees (including time-of-use) offer the best incentive for PV panel and battery deployment. However, this leads to the highest inequalities between consumers and prosumers in terms of electricity costs and, thereby, to cross-subsidies from the former to the latter.

- Strategies based on capacity fees promote the integration of storage devices as they take action to limit the peak of consumption of prosumers, leading once again to a cost distribution between consumers and prosumers where the former bear most of the network costs.

- Strategies based on fixed fees significantly limit the incentives for DER deployment (PV or battery), therefore they hardly show any impact on the distribution of grid costs.

Furthermore, the sensitivity of the model to several input parameters -the Bernoulli bias $\alpha$, the selling price of electricity for prosumers, and the technology price- has been reported, showing that the trends remain constant when applying different values to the parameters, only changing in the rate at which DER installations are deployed and electricity prices increase.

This paper has focused on the regulatory challenges induced by the deployment of distributed power generation, but future works may include modelling physical constraints induced by the distribution network, such as over-voltages or new uses for electricity. Another dimension where this work can be expanded is the possibility of introducing changes in the topology of the network. These changes may aim to introduce future investments in infrastructure depending on the amount of DER installations deployed within the distribution network. Finally, introducing limits on imports and exports from prosumers can be considered in a future expansion of this framework, thus limiting PV deployment in an even more realistic fashion.

\section{Declaration of Competing Interest}

The authors declare that they have no known competing financial interests or personal relationships that could have appeared to influence the work reported in this paper.

\section{References}

[1] Teske S. Achieving the Paris climate agreement goals. Springer; 2019.

[2] Goldthau A. Rethinking the governance of energy infrastructure: Scale, decentralization and polycentrism. Energy Res Soc Sci 2014;1:134-40.

[3] Connolly D, Lund H, Mathiesen B. Smart energy Europe: The technical and economic impact of one potential 100\% renewable energy scenario for the european union. Renew Sustain Energy Rev 2016;60:1634-53.

[4] Lopes JP, Hatziargyriou N, Mutale J, Djapic P, Jenkins N. Integrating distributed generation into electric power systems: A review of drivers, challenges and opportunities. Electric Power Syst Res 2007;77(9):1189-203.

[5] Cossent R, Gómez T, Frías P. Towards a future with large penetration of distributed generation: Is the current regulation of electricity distribution ready? regulatory recommendations under a european perspective. Energy Policy 2009;37(3): $1145-55$.

[6] Lu L, Waddams Price C. Designing distribution network tariffs that are fair for different consumer groups, Tech. rep., University of East Anglia, Centre for Competition Policy, UK; 2018.

[7] Brown T, Faruqui A, Lessem N. Electricity distribution network tariffs: Principles and analysis of options, Tech. rep., The Brattle Group, Boston, MA, USA; 2018.

[8] Council of European Energy Regulators, Electricity distribution network tariffs ceer guidelines of good practice C16-DS-27-03.

[9] Abdelmotteleb I, Gómez T, Ávila JPC, Reneses J. Designing efficient distribution network charges in the context of active customers. Appl Energy 2018;210:815-26.

[10] Eid C, Guillén JR, Marín PF, Hakvoort R. The economic effect of electricity netmetering with solar PV: Consequences for network cost recovery, cross subsidies and policy objectives. Energy Policy 2014;75:244-54.

[11] Gautier A, Jacqmin J, Poudou J-C. The prosumers and the grid. J Regul Econ 2018; 53(1):100-26.

[12] Gautier A, Jacqmin J. Pv adoption: the role of distribution tariffs under net metering. J Regulat Econ 2020;57(1):53-73.

[13] Costello KW, Hemphill RC. Electric utilities "death spiral": hyperbole or reality? Electr J 2014;27(10):7-26.

[14] Simshauser P. Distribution network prices and solar PV: Resolving rate instability and wealth transfers through demand tariffs. Energy Econ 2016;54:108-22.

[15] Manuel de Villena Millan M, Gautier A, Fonteneau R, Ernst D. A multi-agent system approach to model the interaction between distributed generation deployment and the grid. In: Proc of CIRED Workshop 2018; 2018.

[16] Clastres C, Percebois J, Rebenaque O, Solier B. Cross subsidies across electricity network users from renewable self-consumption. Util Policy 2019;59:100925.

[17] Ansarin M, Ghiassi-Farrokhfal Y, Ketter W, Collins J. The economic consequences of electricity tariff design in a renewable energy era. Appl Energy 2020;275: 115317.

[18] Pérez-Arriaga I, Knittel C, et al., Utility of the future, Tech. rep., Massachusetts Institute of Technology, MIT Energy Initiative, Cambridge, MA, USA; 2016. 
[19] Frías P, Gómez T, Cossent R, Rivier J. Improvements in current european network regulation to facilitate the integration of distributed generation. Int J Electr Power Energy Syst 2009;31(9):445-51.

[20] Sotkiewicz PM, Vignolo JM. Towards a cost causation-based tariff for distribution networks with DG. IEEE Trans Power Syst 2007;22(3):1051-60.

[21] Román J, Gómez T, Muñoz A, Peco J. Regulation of distribution network business. IEEE Trans Power Deliv 1999;14(2):662-9.

[22] de Montigny M, Heniche A, Kamwa I, Cauchon L, Mailhot R, Lebeau S, et al. Multiagent stochastic simulation of minute-to-minute grid operations and control to integrate wind generation under AC power flow constraints. IEEE Trans Sustain Energy 2013;4(3):619-29.

[23] Divényi D, Dán AM. Agent-based modeling of distributed generation in power system control. IEEE Trans Sustain Energy 2013;4(4):886-93.

[24] Huang Y, Söder L. Assessing the impact of incentive regulation on distribution network investment considering distributed generation integration. Int J Electr Power Energy Syst 2017;89:126-35.

[25] Siano P. Assessing the impact of incentive regulation for innovation on res integration. IEEE Trans Power Syst 2014;29(5):2499-508.

[26] Degefa MZ, Alahäivälä A, Kilkki O, Humayun M, Seilonen I, Vyatkin V, et al. MAS based modeling of active distribution network: the simulation of emerging behaviors. IEEE Trans Smart Grid 2016;7(6):2615-23.

[27] Schittekatte T, Momber I, Meeus L. Future-proof tariff design: recovering sunk gric costs in a world where consumers are pushing back. Energy Econ 2018;70:484-98.
[28] Schittekatte T, Meeus L Least-cost distribution network tariff design in theory and practice. The Energy Journal 41.

[29] Picciariello A, Alvehag K, Söder L. Impact of network regulation on the incentive for DG integration for the DSO: opportunities for a transition toward a smart grid. IEEE Trans Smart Grid 2015;6(4):1730-9.

[30] Manuel de Villena M, Fonteneau R, Gautier A, Ernst D. Evaluating the evolution of distribution networks under different regulatory frameworks with multi-agent modelling. Energies 2019;12(7):1203.

[31] Quoilin S, Kavvadias K, Mercier A, Pappone I, Zucker A. Quantifying selfconsumption linked to solar home battery systems: Statistical analysis and economic assessment. Appl Energy 2016;182:58-67.

[32] NREL, Crest. https://financere.nrel.gov/finance/content/crest-cost-energy-models.

[33] Stein JS. The photovoltaic performance modeling collaborative (PVPMC). In: 2012 38th IEEE Photovoltaic Specialists Conference, IEEE; 2012. p. 003048-003052.

[34] Manuel de Villena M, Jacqmin J, Fonteneau R, Gautier A, Ernst D. Network tariffs and the integration of prosumers: the case of wallonia, Author pre-print. http:// hdl.handle.net/2268/251102.

[35] Bartusch C, Wallin F, Odlare M, Vassileva I, Wester L. Introducing a demand-based electricity distribution tariff in the residential sector: Demand response and customer perception. Energy Policy 2011;39(9):5008-25.

[36] European Union. Directive 2018/2001 of the european parliament and of the council of 11 december 2018 on the promotion of the use of energy from renewable sources. Official J European Union 2018;328:82-209. 